\title{
Detection of Septic Arthritis using Meta Heuristic Algorithms
}

Jobin Christ MC $^{1^{*}}$, Lakshmi Narayanan $\mathrm{A}^{1}$ and Rahul Krishnan ${ }^{2}$

${ }^{1}$ Department of Biomedical Engineering, Rajalakshmi Engineering College, Chennai, India

${ }^{2}$ Department of Electronics \& Communication Engineering, Rajalakshmi Institute of Technology, Chennai, India

*Corresponding author: Jobin Christ MC, Professor, Department of Biomedical Engineering, Rajalakshmi Engineering College, Chennai, India, Tel: 0443718 1111; Email: jobinchrist@gmail.com

Received date: November 14, 2017; Accepted date: November 21, 2017; Published date: November 25, 2017

Copyright: @ 2017 Christ MCB, et al. This is an open-access article distributed under the terms of the Creative Commons Attribution License, which permits unrestricted use, distribution, and reproduction in any medium, provided the original author and source are credited.

\begin{abstract}
Arthritis is one of the kinds of chronic disease which causes inflammation to the joints which causing pain and stiffness that can worsen with aging. Mostly it is caused due to the decrease of Cartilage thickness present between the bone joints. There are more than 100 types of arthritis exist in the world. In general arthritis is classified into two parts, septic arthritis and reactive arthritis. Septic arthritis is an unfavorable arthropathy originated by an intraarticular infection which is usually connected to severe symptoms such as pain and decreased the range of motion. In this work, an analysis has been done on two meta-heuristic methods for early detection of septic arthritis since it is a direct invasion of bacteria. In this paper two different meta-heuristic methods like Ant Colony Optimization (ACO) and Clown Fish Queuing and Switching Optimization Algorithm (CFQSOA) are analyzed for the early detection of septic arthritis. By the early diagnosis and treatment of arthritis, the damage to the joints can be reduced.
\end{abstract}

Keywords: Arthritis; Septic Arthritis; Meta Heuristic Algorithms; Image Processing

\section{Introduction}

Arthritis is the common disease among elderly people that usually happens between the bone joints where the cartilages are present. Due to the continuous usage of the joints, this cartilage will be reduced gradually, and at a certain level, it will be disappeared. Then the bones will encounter each other which results in severe pain. Arthritis causes deformation in joints and leads to incapacitation in motion. Septic arthritis is a kind of painful infection of a joint. The infection can occur from germs that travel through your bloodstream from another part of your body. There is a chance to occur Septic arthritis when a penetrating injury supplies germs straight into the joint. Infants and older adults are usually feasible to develop septic arthritis. Knees are the hotspot for arthritis, but septic arthritis also can affect hips, shoulders and other joints. The infection can spread and severely affect the cartilage and bone inside the joint, so prompt treatment is very important. Treatment involves draining the joint with a needle or surgically [1]. The diagnosis of joint sepsis is mostly straightforward because the victim often presents with a painful joint, purulent synovial fluid and suffered by fever. The main influence that contributes to septic arthritis comprises bacteraemia, old age, prosthetic joints, an immune compromised state and intra-articular injections. Imaging generally plays an adjunct role to arthrocentesis in the diagnosis of joint sepsis. If synovial fluid cannot be retrieved, it cannot be reverted and causes impairment in motion. Thus early detection of arthritis may help to the reduction of the damage to the joints [2]. Here, radiology plays a major role here. X-ray, CT, MRI and Ultrasound methods are used commonly for the early diagnosis of septic arthritis. However various image processing algorithms will help the radiologists to diagnose septic arthritis in a faster manner.

In this work two different meta-heuristic methods such as Ant Colony Optimization (ACO) and Clown Fish Queuing and Switching
Optimization Algorithm (CFQSOA) are analyzed for the early detection of septic arthritis. The use of meta-heuristics has significantly increased the ability of finding very high-quality solutions to hard, practically relevant combinatorial optimization problems in a reasonable time [3].

\section{Ant Colony Optimization}

Ant Colony Optimization (ACO) is a popular population based optimization method [4]. ACO is based on the inspiration of real ant colony and their combined summing behavior. Real ants are having the capacity of finding the shortest route from a food source to their nest without using visual indications. In general ants will move on a straight line that connects the food source to their nest is a pheromone trail [5]. Pheromone is a volatile chemical substance produced by ants while moving, and each ant probabilistically prefers to follow a direction rich in pheromone [6]. This behavioral aspect of the real ants may be helpful to obtain optimized value from a population. In ACO, each and every ant builds a part of the solution using an artificial pheromone, the pheromone which is having high value that will influence in the results.

\section{Clown Fish Queuing and Switching Optimization Algorithm}

This algorithm is based on the clown fish's queuing characteristics. The clown fish is a type of fish; they attach among themselves and also survive symbiotically with the other sea anemones. The clown fish's ecological mannerisms are to obtain the social rank in the foremost position in the shorter queues in sea anemone. The female clown fish are mostly dominant and large in size. The male clown fish are small in size. Some sub ordinates and immature clown fishes also be there. If female fish removed or rotten, the male fishes get that position with shortest queues. Now the male becomes dominant one and the sub ordinate will become new male to acquire their social ranks in the shorter queues. The basic performances of CFQSOA are based on four 
Citation: Jobin Christ MC, Narayanan AL, Krishnan R (2017) Detection of Septic Arthritis using Meta Heuristic Algorithms. J Arthritis 6: 259. doi:

Page 2 of 2

things; prey, swarm, follow, and shift where they search for foremost position searching. All these behaviors are based on the artificial fish swarm intelligence [7].

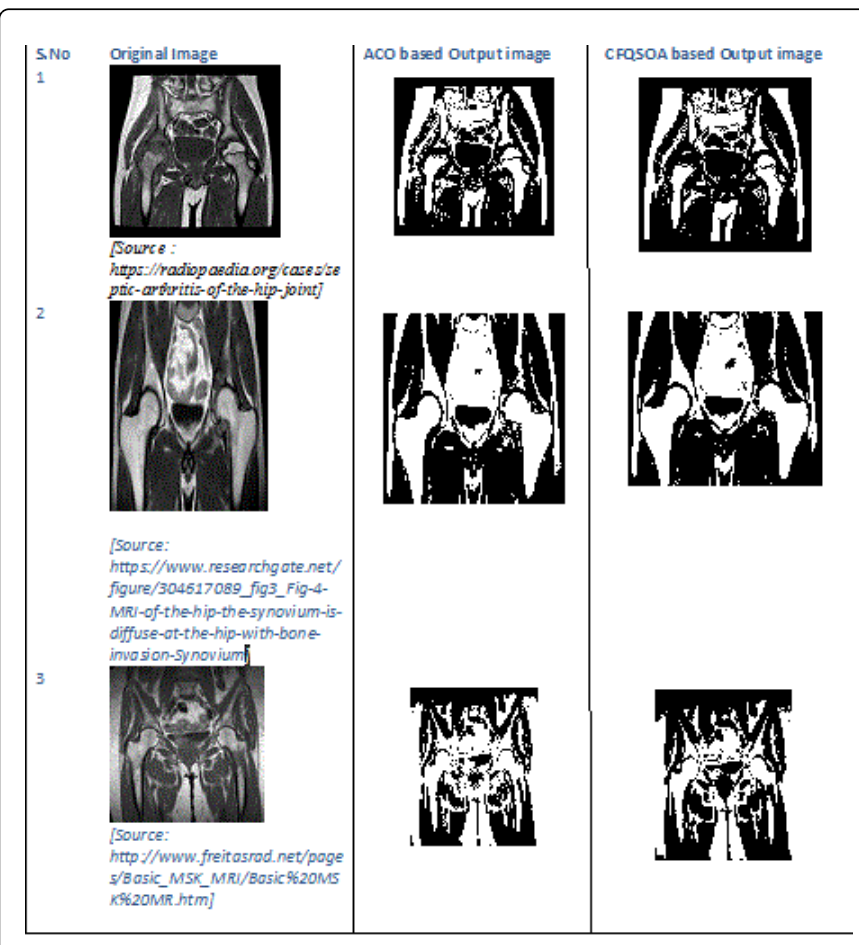

Figure 1: Comparison of Results.

\section{Conclusion}

In this review article two different meta-heuristic methods such as Ant Colony Optimization (ACO) and Clown Fish Queuing and
Switching Optimization Algorithm (CFQSOA) are analyzed for the early detection of septic arthritis. The images used are Septic Arthritis of Hip Joint; the synovium is diffuse at the hip with bone invasion and Acetabularlabral tear with paralabral cyst. The two methods are implemented using MATLAB 7.9.0 (R200 9b). Results of two algorithms are tabulated in Figure 1. By looking of the output images, physician can easily identify and assess cartilage at knee, which will be useful for the prediction of arthritis. Results show that the CFQSOA can perform better than ACO.

\section{References}

1. https://www.mayoclinic.org/diseases-conditions/bone-and-jointinfections/symptoms-causes/syc 20350755

2. https://radiopaedia.org/articles/septic-arthritis

3. Selvanayaki K (2013) Intelligent brain tumor tissue segmentation from magnetic resonance image (mri) using meta heuristic algorithms. Journal of Global Research in Computer Science 4: 13-20.

4. Devos A, Lukas L, Simonetti A, Suykens J, Vanhamme L et al. (2004) Does the combination of magnetic resonance imaging and spectroscopic imaging improve the classification of brain tumours?," in Engineering in Medicine and Biology Society, IEMBS’04. 26th Annual International Conference of the IEEE, India 1: 407-410.

5. Dorigo M, Maniezzo V, Colorni A (1996) Ant system: optimization by a colony of cooperating agents," Systems, Man, and Cybernetics, Part B: Cybernetics. IEEE Transactions 26: 29-41.

6. Anandgaonkar GP, Sable GS (2013) Detection and Identification of Brain Tumor in Brain MR Images Using Fuzzy C-Means Segmentation. International Journal of Advanced Research in Computer and Communication Engineering 2: 3964-3967.

7. Jobin Christ MC, Subramanian R (2016) Clown fish queuing and switching optimization algorithm for brain tumor segmentation. Biomedical Research 27: 65-69. 